\title{
Observational Constraints on Quintessence: Thawing, Tracker, and Scaling models
}

\author{
Takeshi Chiba, ${ }^{1}$ Antonio De Felice, ${ }^{2,3}$ and Shinji Tsujikawa ${ }^{4}$ \\ ${ }^{1}$ Department of Physics, College of Humanities and Sciences, Nihon University, Tokyo 156-8550, Japan \\ ${ }^{2}$ ThEP's CRL, NEP, The Institute for Fundamental Study, \\ Naresuan University, Phitsanulok 65000, Thailand \\ ${ }^{3}$ Thailand Center of Excellence in Physics, Ministry of Education, Bangkok 10400, Thailand \\ ${ }^{4}$ Department of Physics, Faculty of Science, Tokyo University of Science, \\ 1-3, Kagurazaka, Shinjuku-ku, Tokyo 162-8601, Japan
}

(Dated: April 5, 2013)

\begin{abstract}
For two types of quintessence models having thawing and tracking properties, there exist analytic solutions for the dark energy equation of state $w$ expressed in terms of several free parameters. We put observational bounds on the parameters in such scenarios by using the recent data of Supernovae type Ia (SN Ia), Cosmic Microwave Background (CMB), and Baryon Acoustic Oscillations (BAO). The observational constraints are quite different depending on whether or not the recent BAO data from BOSS are taken into account. With the BOSS data the upper bounds of today's values of $w$ $\left(=w_{0}\right)$ in thawing models is very close to -1 , whereas without this data the values of $w_{0}$ away from -1 can be still allowed. The tracker equation of state $w_{(0)}$ during the matter era is constrained to be $w_{(0)}<-0.949$ at $95 \%$ confidence level $(\mathrm{CL})$ even without the BOSS data, so that the tracker models with $w$ away from -1 are severely disfavored. We also study observational constraints on scaling models in which $w$ starts to evolve from 0 in the deep matter era and show that the transition to the equation of state close to $w=-1$ needs to occur at an early cosmological epoch. In the three classes of quintessence models studied in this paper, the past evolution of the Hubble parameters in the best-fit models shows only less than the $2.5 \%$ difference compared to the $\Lambda$ CDM.
\end{abstract}

\section{INTRODUCTION}

Independent observational data such as SN Ia [1, 2], CMB [3, 4], and BAO [5, 6] suggest that about $70 \%$ of the energy density today consists of dark energy responsible for cosmic acceleration. For the constant dark energy equation of state $w$ the recent joint data analysis based on SN Ia, CMB, BAO, and the Hubble constant measurement shows that $w$ is constrained to be $w=-1.013_{-0.073}^{+0.068}$ at $68 \%$ CL [7]. If we use the timedependent parametrization $w(a)=w_{0}+w_{a}(1-a)$, where $a$ is the scale factor normalized as $a=1$ today, the two parameters $w_{0}$ and $w_{a}$ are constrained to be $w_{0}=-1.046_{-0.170}^{+0.179}$ and $w_{a}=0.14_{-0.76}^{+0.60}[$ ].

One of the simplest candidates of dark energy is the cosmological constant characterized by the equation of state $w=-1$, which is consistent with the current observational data. However, if the cosmological constant originates from the vacuum energy associated with particle physics, there is a huge gap between the theoretical and observed values [8]. Instead, alternative dark energy models with dynamically changing $w$ - such as quintessence [9, 10] and k-essence [11] - have been proposed (see Refs. 12] for reviews).

Quintessence is described by a canonical scalar field $\phi$ with a potential $V(\phi)$. In the framework of particle physics it is generally difficult to accommodate a very light scalar field with a mass of the order of the Hubble parameter $H_{0} \approx 10^{-33} \mathrm{eV}$ today 13 , 14. However there have been theoretical attempts to construct viable quintessence models in particle physics - especially in supersymmetric theories [15]. For example, the PseudoNambu-Goldstone-Boson (PNGB) [16] or axions [17] have the potential of the form $V(\phi)=\Lambda^{4}[1 \pm \cos (\phi / f)]$ with suppressed quantum corrections.

Caldwell and Linder [18] classified quintessence models into two classes, depending on the evolution of $w$. The first class corresponds to thawing models, in which the field is nearly frozen by a Hubble friction during the early cosmological epoch and it starts to evolve once the field mass $m_{\phi}$ drops below the Hubble rate $H$. In this case the evolution of $w$ is characterized by the growth from -1 . The representative potential of this class is the hilltop potential such as $V(\phi)=\Lambda^{4}[1+\cos (\phi / f)]$.

The second class consists of freezing models, in which the evolution of the field gradually slows down because of the shallowness of the potential at late times. For the inverse power-law potential $V(\phi)=M^{4+p} \phi^{-p}(p>0)$ [10] there is a so-called tracker solution characterized by a nearly constant field equation of state $w=-2 /(p+2)$ during the matter era 19]. In this case the solutions with different initial conditions approach a common trajectory (tracker) first, which is followed by the decrease of $w$ toward -1 .

In addition to tracking freezing models there is another sub-class of freezing models associated with scaling solutions [20]. In this case the field equation of state scales as that of the background fluid during most of the matter era $(w \approx 0)$. The representative potential of this class is $V(\phi)=V_{1} e^{-\lambda_{1} \phi / M_{\mathrm{pl}}}+V_{2} e^{-\lambda_{2} \phi / M_{\mathrm{pl}}}$, where $M_{\mathrm{pl}}$ is the reduced Planck mass, $\lambda_{1}$ and $\lambda_{2}$ are constants with $\lambda_{1} \gg 1$ and $\lambda_{2} \lesssim 1$ [21]. In the early matter era the potential is approximated as $V(\phi) \simeq V_{1} e^{-\lambda_{1} \phi / M_{\mathrm{pl}}}$, which gives rise to the scaling solution characterized by $w=0$ with the field density parameter $\Omega_{\phi}=3 / \lambda_{1}^{2}$. At late times the dominance of the potential $V_{2} e^{-\lambda_{2} \phi / M_{\mathrm{pl}}}$ leads to the rapid de- 
crease of $w$ relative to the tracker case mentioned above. Note that the potential $V(\phi)=e^{-\lambda \phi / M_{\mathrm{pl}}}\left[(\phi-B)^{\alpha}+A\right]$ proposed in Ref. 22 also exhibits a similar property to that of the double exponential potential (see also Ref. 23]).

In this paper we place observational constraints on three types of quintessence models: (i) thawing models, (ii) tracking freezing models, and (iii) scaling freezing models (see Refs. 24] for related works). This analysis covers most of quintessence potentials proposed in literature.

For thawing models we employ the analytic expression of $w$ derived in Ref. [25, 26] under the approximation that $|w+1| \ll 1$ (see also Ref. [27]). The likelihood analysis with the SN Ia and BAO data was carried out in Ref. 28] (see also Ref. 25]). We update the analysis by using the latest SN Ia data (Union 2.1 dataset [7]) and by adding the data of CMB shift parameters measured by WMAP7 [4]. We also take into account the recent BAO data of the BOSS experiment [29]. ${ }^{1}$

Note that the observational constraints on thawing models were carried out by using a multi-parameter extension of the exponential potential [31] and by introducing a statefinder hierarchy [32]. Our study based on the analytic solution of $w$ is more convenient in that it covers any quintessence potential having thawing properties and that $w$ is expressed in terms of three parameters without the need of introducing more free parameters.

For tracking freezing models one of the present authors obtained the approximate analytic formula of $w$ expressed in terms of two free parameters [33] (see also Ref. 34]). The likelihood analysis based on the SN Ia and $\mathrm{BAO}$ data was performed in Ref. [33]. We show that adding the CMB and BOSS data further strengthens the constraints on the tracker equation of state $w_{(0)}$. Wang et al. [35] placed observational bounds on a number of quintessence potentials having tracker properties. Our study based on the analytic formula of $w$ is general enough to cover such potentials. Moreover we show that inclusion of the BOSS BAO data further strengthens the bounds on $w_{(0)}$ previously derived in the literature.

For scaling freezing models it is difficult to derive an analytic expression of $w$, so we resort to numerical simulations to find a viable parameter space.

This paper is organized as follows. In Sec. II we briefly review the procedure to derive approximate analytic expressions of $w$ in thawing and tracking freezing models. The accuracy of those approximations is also discussed by solving the equations of motion numerically. For scaling freezing models we show that in some cases it is possible to fit the evolution of $w$ by using a specific parametrization. In Sec. III we first explain the method of our likeli-

\footnotetext{
1 Two months after the initial submission of this paper, new $\mathrm{BAO}$ data in the Ly $\alpha$ forest appeared in the redshift range $2.1 \leq z \leq 3.5[30]$. We do not take into account this new data in our likelihood analysis.
}

hood analysis based on the SN Ia, CMB, and BAO data and then we proceed to observational constraints on three classes of quintessence models. Sec. IV is devoted to conclusions.

\section{PARAMETRIZATIONS OF QUINTESSENCE}

Quintessence [9] is described by a minimally coupled scalar field $\phi$ with a potential $V(\phi)$. In addition to the field $\phi$ we take into account non-relativistic matter with an energy density $\rho_{m}$. The action in such a system is given by

$$
S=\int d^{4} x \sqrt{-g}\left[\frac{M_{\mathrm{pl}}^{2}}{2} R-\frac{1}{2} g^{\mu \nu} \partial_{\mu} \phi \partial_{\nu} \phi-V(\phi)\right]+S_{m},
$$

where $g$ is the determinant of the metric $g_{\mu \nu}, R$ is the Ricci scalar, and $S_{m}$ is the action for non-relativistic matter. In the flat Friedmann-Lemaitre-Robertson-Walker background with the scale factor $a(t)$, the dynamical equations of motion are

$$
\begin{aligned}
& 3 H^{2} M_{\mathrm{pl}}^{2}=\rho_{\phi}+\rho_{m}, \\
& \ddot{\phi}+3 H \dot{\phi}+V_{, \phi}=0, \\
& \dot{\rho}_{m}+3 H \rho_{m}=0,
\end{aligned}
$$

where $H=\dot{a} / a$ is the Hubble parameter, a dot represents a derivative with respect to cosmic time $t, \rho_{\phi}=\dot{\phi}^{2} / 2+$ $V(\phi)$, and $V_{, \phi}=d V / d \phi$. The pressure of the field is given by $P_{\phi}=\dot{\phi}^{2} / 2-V(\phi)$. We introduce the equation of state $w=P_{\phi} / \rho_{\phi}$ and the density parameter $\Omega_{\phi}=$ $\rho_{\phi} /\left(3 H^{2} M_{\mathrm{pl}}^{2}\right)$ of dark energy.

From Eqs. (2)-(4) we obtain the following equations for $w$ and $\Omega_{\phi}[25$, 27] (see also Refs. [20, 36]):

$$
\begin{aligned}
w^{\prime} & =(1-w)\left[-3(1+w)+\lambda \sqrt{3(1+w) \Omega_{\phi}}\right], \\
\Omega_{\phi}^{\prime} & =-3 w \Omega_{\phi}\left(1-\Omega_{\phi}\right) \\
\lambda^{\prime} & =-\sqrt{3(1+w) \Omega_{\phi}}(\Gamma-1) \lambda^{2} .
\end{aligned}
$$

where $\lambda=-M_{\mathrm{pl}} V_{, \phi} / V, \Gamma=V V_{, \phi \phi} / V_{, \phi}^{2}$, and a prime represents a derivative with respect to $N^{\phi}=\ln a$. Depending on the field potential and the initial conditions, there are several different cases for the evolution of $w$ [18].

In the following we shall discuss possible analytic solutions of $w$ for three different cases: (i) thawing, (ii) tracking freezing, and (iii) scaling freezing models. The presence of analytic solutions is useful in that some general properties of physical parameters can be extracted without studying a host of quintessence potentials separately. Moreover, if $w$ can be analytically expressed in terms of the redshift $z$ with several free parameters, we do not need to integrate the background equations of motion with arbitrary initial conditions. This greatly simplifies the likelihood analysis carried out in Sec. III. 


\section{A. Thawing models}

For thawing models of quintessence the field $\phi$ is nearly frozen in the early matter era because of the Hubble friction, so that $w$ is close to -1 . One can regard $w=-1$ as the fixed point of Eq. (5). For $\lambda \neq 0$ such a point is not stable, and hence $w$ starts to grow at the late cosmological epoch.

If we assume that $\lambda$ is nearly constant, one can express $w$ in terms of $\Omega_{\phi}$ by using Eqs. (5) and (6) under the approximation $|1+w| \ll 1$ [27]. This neglects the effect of the field mass squared $V_{, \phi \phi}$, but it is possible to derive a more elaborate form of $w$ with the mass term taken into account [25, 26]. In doing so, the potential is expanded around the initial field value $\phi_{i}$ up to second order, i.e., $V(\phi)=\sum_{n=0}^{2} V^{(n)}\left(\phi_{i}\right)\left(\phi-\phi_{i}\right)^{n} / n !$.

Provided that $|w+1| \ll 1$, the evolution of the scale factor can be approximated by that of the $\Lambda$ CDM model, i.e., $a(t)=\left[\left(1-\Omega_{\phi 0}\right) / \Omega_{\phi 0}\right]^{1 / 3} \sinh ^{2 / 3}\left(t / t_{\Lambda}\right)$, where $t_{\Lambda}=$ $2 M_{\mathrm{pl}} / \sqrt{3 V\left(\phi_{i}\right)}$ and $\Omega_{\phi 0}$ is the today's density parameter of quintessence. On using this solution, Eq. (3) is integrated to give the field in terms of the function of $t$ and hence the field equation of state $w(t) \simeq-1+\dot{\phi}^{2} / V\left(\phi_{i}\right)$ is known. This process leads to the following analytic expression of $w$ [25, 26]:

$$
w(a)=-1+\left(1+w_{0}\right) a^{3(K-1)}\left[\frac{(K-F(a))(F(a)+1)^{K}+(K+F(a))(F(a)-1)^{K}}{\left(K-\Omega_{\phi 0}^{-1 / 2}\right)\left(\Omega_{\phi 0}^{-1 / 2}+1\right)^{K}+\left(K+\Omega_{\phi 0}^{-1 / 2}\right)\left(\Omega_{\phi 0}^{-1 / 2}-1\right)^{K}}\right]^{2},
$$

where $w_{0}$ is the value of $w$ today, and

$$
\begin{aligned}
K & =\sqrt{1-\frac{4 M_{\mathrm{pl}}^{2} V_{, \phi \phi}\left(\phi_{i}\right)}{3 V\left(\phi_{i}\right)}}, \\
F(a) & =\sqrt{1+\left(\Omega_{\phi 0}^{-1}-1\right) a^{-3}} .
\end{aligned}
$$

The solution (8) is valid for $K^{2}>0$. The equation of state (8) is expressed in terms of the three parameters $w_{0}, \Omega_{\phi 0}$, and $K$.

As a concrete example, let us consider the hilltop potential

$$
V(\phi)=\Lambda^{4}[1+\cos (\phi / f)] .
$$

In this case the parameter (9) is given by

$$
K=\left[1+\frac{4}{3}\left(\frac{M_{\mathrm{pl}}}{f}\right)^{2} \frac{\cos \left(\phi_{i} / f\right)}{1+\cos \left(\phi_{i} / f\right)}\right]^{1 / 2} .
$$

If $0<\phi_{i} / f<\pi / 2$ and $\pi / 2<\phi_{i} / f<\pi$, one has $K>1$ and $K<1$ respectively. In the former case the potential is approximately given by $V(\phi) \approx 2 \Lambda^{4}\left[1-\phi^{2} /\left(4 f^{2}\right)\right]$ around $\phi=0$, whereas in the latter case it is approximated as $V(\phi) \approx \Lambda^{4}(\phi-\pi f)^{2} /\left(2 f^{2}\right)$ around $\phi=\pi f$.

In Fig. 1 we plot the numerical evolution of $w$ versus $a$ for $K>1$ with several different values of $f$ and $\phi_{i}$. The bald dashed curves correspond to the results derived by the analytic expression (8), which show good agreement with the numerically integrated solutions for $w_{0} \lesssim-0.3$. For $K$ larger than 10 , the initial displacement of the field is required to be close to 0 to avoid its rapid roll down. In such cases the field mass is largely negative, which leads to the tachyonic instability of field perturbations. If the field reaches the potential minimum

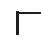

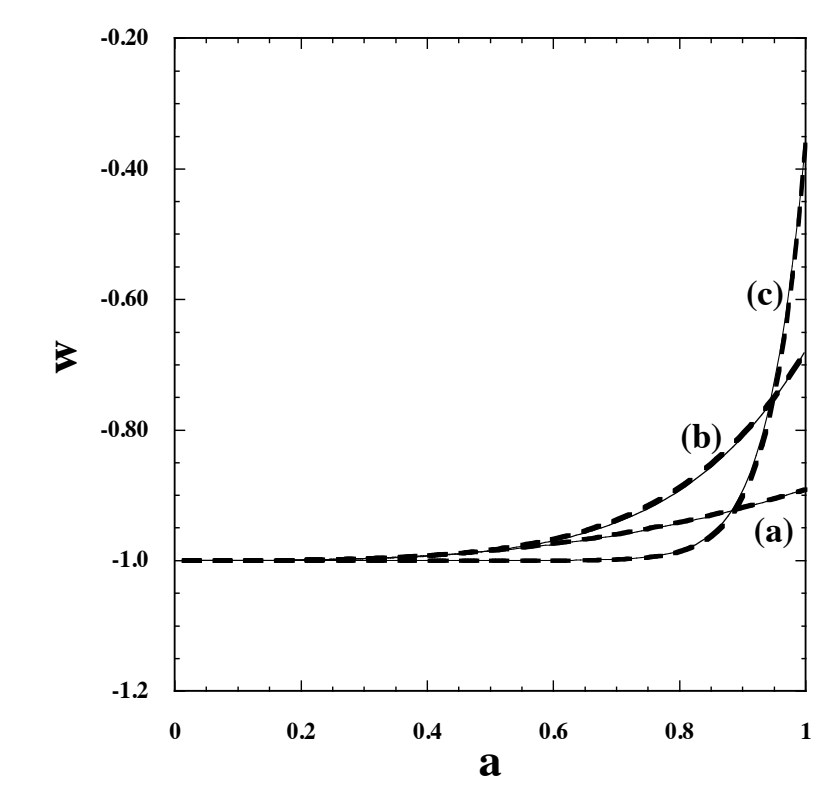

Figure 1: The quintessence equation of state $w$ versus $a$ for the potential (11) with (a) $f / M_{\mathrm{pl}}=0.5, \phi_{i} / f=0.5(K=1.9)$, (b) $f / M_{\mathrm{pl}}=0.3, \phi_{i} / f=0.25(K=2.9)$, and (c) $f / M_{\mathrm{pl}}=0.1$, $\phi_{i} / f=7.6 \times 10^{-4}(K=8.2)$. These cases correspond to $V_{, \phi \phi}\left(\phi_{i}\right)<0$, so that $K>1$. The solid curves show numerical solutions, whereas the bald dashed curves describe the results derived from the parametrization (8) with $\Omega_{\phi 0}=0.73$.

by today and it starts to oscillate, numerical simulations show that Eq. (8) is no longer reliable. We set the prior $K<10$ in the likelihood analysis of Sec. IIIA.

The analytic estimation (8) starts to lose accuracy for $K$ smaller than 1 . This reflects the fact that the field is initially located away from the potential maximum. 
Then the Taylor expansion around $\phi=\phi_{i}$ tends to be more inaccurate because of the rapid variation of the field. Numerically we find that the analytic solution (8) is reliable for $0.5 \lesssim K<1$ and $w_{0} \lesssim-0.8$.

\section{B. Tracking freezing models}

In Eq. (5) there is another fixed point given by

$$
\Omega_{\phi}=\frac{3(1+w)}{\lambda^{2}}
$$

along which $w$ is constant. This corresponds to the tracker that attracts the solutions with different initial conditions to a common trajectory. The condition under which the tracking occurs is $[19]^{2}$

$$
\Gamma>1
$$

In this case the variable $\lambda$ approaches 0 .

From (13) it follows that $\Omega_{\phi}^{\prime} / \Omega_{\phi}=-2 \lambda^{\prime} / \lambda$. Using this relation with Eqs. (6) and (7) in the regime $\Omega_{\phi} \ll 1$, the field equation of state along the tracker is given by

$$
w=w_{(0)} \equiv-\frac{2(\Gamma-1)}{2 \Gamma-1} .
$$

For the potential $V(\phi)=M^{4+p} \phi^{-p}(p>0)$ one has $\Gamma=1+1 / p$ and hence $w_{(0)}=-2 /(p+2)[19,34]$.

The result (15) was derived by neglecting the contribution of $\Omega_{\phi}$, but its effect can be accommodated by dealing with $\Omega_{\phi}$ as a perturbation to the 0 -th order solution (15) [33]. We consider the first-order perturbation $\delta w$ around $w_{(0)}$ and then approximate $\Omega_{\phi}(a)$ by the 0 -th order solution

$$
\Omega_{\phi}(a)=\frac{\Omega_{\phi 0} a^{-3 w_{(0)}}}{\Omega_{\phi 0} a^{-3 w_{(0)}}+1-\Omega_{\phi 0}} .
$$

For the models in which $\Gamma$ is nearly constant, we obtain the following analytic solution [33]:

$$
\begin{aligned}
w(a)= & w_{(0)}+\sum_{n=1}^{\infty} \frac{(-1)^{n-1} w_{(0)}\left(1-w_{(0)}^{2}\right)}{1-(n+1) w_{(0)}+2 n(n+1) w_{(0)}^{2}}\left(\frac{\Omega_{\phi}(a)}{1-\Omega_{\phi}(a)}\right)^{n} \\
= & w_{(0)}+\frac{\left(1-w_{(0)}^{2}\right) w_{(0)}}{1-2 w_{(0)}+4 w_{(0)}^{2}} \Omega_{\phi}(a)+\frac{\left(1-w_{(0)}^{2}\right) w_{(0)}^{2}\left(8 w_{(0)}-1\right)}{\left(1-2 w_{(0)}+4 w_{(0)}^{2}\right)\left(1-3 w_{(0)}+12 w_{(0)}^{2}\right)} \Omega_{\phi}(a)^{2} \\
& +\frac{2\left(1-w_{(0)}^{2}\right) w_{(0)}^{3}\left(4 w_{(0)}-1\right)\left(18 w_{(0)}+1\right)}{\left(1-2 w_{(0)}+4 w_{(0)}^{2}\right)\left(1-3 w_{(0)}+12 w_{(0)}^{2}\right)\left(1-4 w_{(0)}+24 w_{(0)}^{2}\right)} \Omega_{\phi}(a)^{3}+\cdots,
\end{aligned}
$$

where in the second and third lines we carried out the expansion $\left(\Omega_{\phi}(a) /\left(1-\Omega_{\phi}(a)\right)\right)^{n}=$ $\Omega_{\phi}(a)^{n}\left(\sum_{m=0}^{\infty} \Omega_{\phi}(a)^{m}\right)^{n}$. The equation of state (18) is expressed in terms of the two parameters $w_{(0)}$ and $\Omega_{\phi 0}$. Numerically we confirm that the approximated formula (18) tends to approach the full numerical solution by adding higher-order terms of $\Omega_{\phi}(a)$.

\section{Scaling freezing models}

The scaling solution [20] is a special case of a tracker along which $\Omega_{\phi}$ in Eq. (13) is constant with $0<\Omega_{\phi}<1$. From Eq. (6) it then follows that $w=0$ and hence $\Omega_{\phi}=$ $3 / \lambda^{2}$ during the matter era. Since $\lambda$ is constant, one has $\Gamma=1$ from Eq. (7). This corresponds to the exponential potential $V(\phi)=V_{0} e^{-\lambda \phi / M_{\mathrm{pl}}}$, where $V_{0}$ is a constant ${ }^{3}$. In this case, however, the field equation of state is the same as that of the background fluid $(w=0)$, so that the system does not enter the phase of cosmic acceleration.

This problem can be circumvented for the following model 21]

$$
V(\phi)=V_{1} e^{-\lambda_{1} \phi / M_{\mathrm{pl}}}+V_{2} e^{-\lambda_{2} \phi / M_{\mathrm{pl}}},
$$

where $\lambda_{i}$ and $V_{i}(i=1,2)$ are constants. If $\lambda_{1} \gg 1$ and $\lambda_{2} \lesssim 1$ then the solution first enters the scaling regime characterized by $\Omega_{\phi}=3\left(1+w_{m}\right) / \lambda_{1}^{2}$, where $w_{m}$ is the equation of state of the background fluid. From the

\footnotetext{
3 For the k-essence Lagrangian $P(\phi, X)$ (where $X=$ $\left.-g^{\mu \nu} \partial_{\mu} \phi \partial_{\nu} \phi / 2\right)$ the condition for the existence of scaling solutions restricts the Lagrangian in the form $P=X g(Y)$, where $g$ is an arbitrary function in terms of $Y=X e^{\lambda \phi / M_{\mathrm{pl}}}$ [38]. Quintessence with the exponential potential corresponds to the choice $g(Y)=1-V_{0} / Y$.
} 


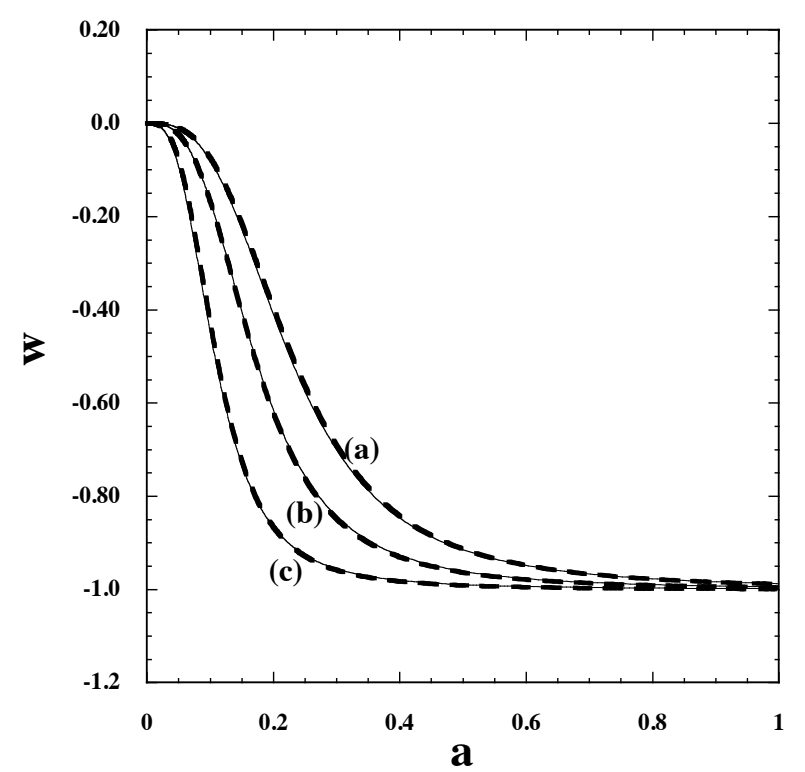

Figure 2: The quintessence equation of state $w$ versus $a$ for the potential (19) with (a) $\lambda_{1}=10, \lambda_{2}=0$, (b) $\lambda_{1}=15$, $\lambda_{2}=0$, and (c) $\lambda_{1}=30, \lambda_{2}=0$. The solid curves show the numerical solutions, whereas the dashed curves represent the results derived from the parametrization (20) with $w_{p}=0$ and $w_{f}=-1$. Each dashed curve corresponds to (a) $a_{t}=0.23$, $\tau=0.33$, (b) $a_{t}=0.17, \tau=0.33$, and (c) $a_{t}=0.11, \tau=0.32$.

bound coming from big bang nucleosynthesis 39] there is a constraint $\Omega_{\phi}<0.045(95 \% \mathrm{CL})$ during the radiation era $\left(w_{m}=1 / 3\right)$, which translates into the condition $\lambda_{1}>9.4$. The scaling matter era $\left(\Omega_{\phi}=3 / \lambda_{1}^{2}, w=0\right)$ is followed by the dark energy dominated epoch driven by the presence of the potential $V_{2} e^{-\lambda_{2} \phi / M_{\mathrm{pl}}}$. If $\lambda_{2}^{2}<3$, the solution approaches another attractor characterized by $\Omega_{\phi}=1$ and $w=-1+\lambda_{2}^{2} / 3$ [20]. The cosmic acceleration occurs for $\lambda_{2}^{2}<2$.

The onset of the transition from the scaling matter era to the dark energy dominated epoch depends on the parameters $\lambda_{1}, \lambda_{2}$, and $V_{2} / V_{1}$. Numerically we find that the transition redshift is not very sensitive to the choice of $V_{2} / V_{1}$, so we study the case $V_{2}=V_{1}$. In Fig. 2 we plot the numerical evolution of $w$ for $\lambda_{2}=0$ with three different values of $\lambda_{1}$. For larger $\lambda_{1}$ the transition to the dark energy dominated epoch occurs earlier.

It is possible to accommodate the above variation of $w$ analytically by using the parametrization proposed by Linder and Huterer [40] (see also Refs. [41]):

$$
w(a)=w_{f}+\frac{w_{p}-w_{f}}{1+\left(a / a_{t}\right)^{1 / \tau}},
$$

where $w_{p}$ and $w_{f}$ are asymptotic values of $w$ in the past and future respectively, $a_{t}$ is the scale factor at the transition, and $\tau$ describes the width of the transition. The scaling solution during the matter era corresponds to $w_{p}=0$. For $\lambda_{2}=0$ one has $w_{f}=-1$, so that the parametrization (20) reduces to $w(a)=-1+[1+$

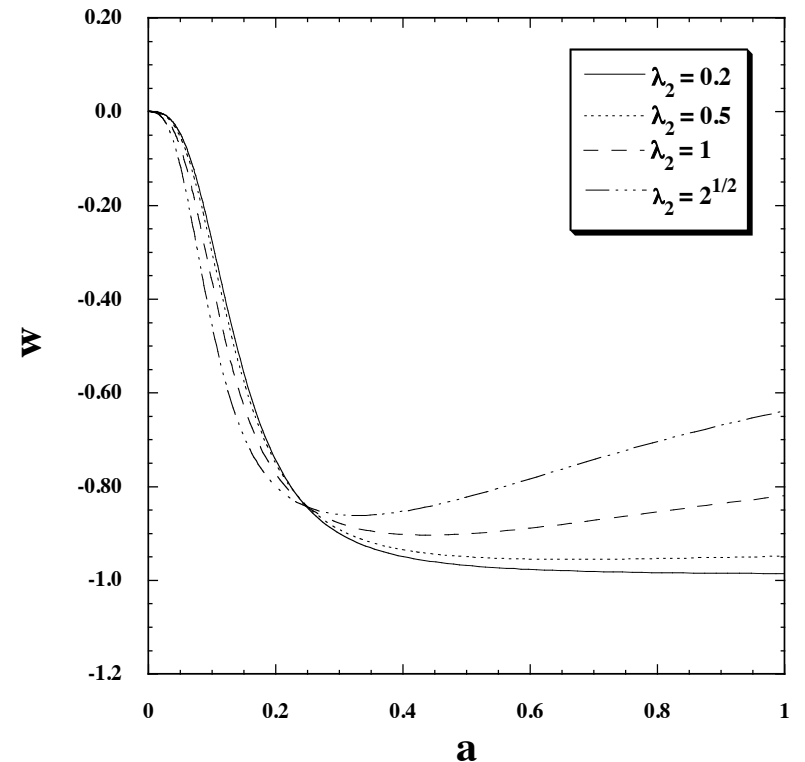

Figure 3: The quintessence equation of state $w$ versus $a$ for the potential (19) with $\lambda_{1}=20$ and $\lambda_{2}=0.2,0.5,1, \sqrt{2}$.

$\left.\left(a / a_{t}\right)^{1 / \tau}\right]^{-1}$. Figure 2 shows that the parametrization (20) can fit the numerical evolution of $w$ very well for appropriate choices of $a_{t}$ and $\tau$. For $\lambda_{2}=0$ the transition width is around $\tau \approx 0.33$, while $a_{t}$ depends on the values of $\lambda_{1}$. In this case one can carry out the likelihood analysis by fixing $\tau=0.33$ and find the constraints on $a_{t}$ (see Ref. [42] for a related work).

If $\lambda_{2} \neq 0$, then the field equation of state finally approaches the value $w=-1+\lambda_{2}^{2} / 3$. Numerically we find that $w$ tends to have a minimum for larger $\lambda_{2}$ before the solutions reach the attractor. If $\lambda_{1}=20$, for example, the minimum appears for $\lambda_{2} \gtrsim 0.3$ (see Fig. 33). In order to place observational bounds on $\lambda_{2}$ in such cases, we need to resort to numerical simulations without using the parametrization (20).

\section{OBSERVATIONAL CONSTRAINTS}

In this section we place observational constraints on the three types of quintessence models separately. We use the recent SN Ia data called Union 2.1 dataset [7], shift parameters provided by WMAP7 [4], and the BAO distance measured by SDSS7 [6] and by BOSS [29]. In order to make the analysis simpler, we fix, for all the models under consideration, the today's radiation density parameter to be equal to the one of the $\Lambda$ CDM model.

In SN Ia observations the luminosity distance $d_{L}(z)=$ $(1+z) \int_{0}^{z} H^{-1}(\tilde{z}) d \tilde{z}$ is measured by the difference (distance modulus) of the apparent magnitude $m(z)$ and the absolute magnitude $M$, as

$$
\mu(z) \equiv m(z)-M=5 \log _{10}\left[d_{L}(z) / 10 \mathrm{pc}\right] .
$$


For the observed distance modulus $\mu_{\mathrm{obs}}\left(z_{i}\right)$ with the errors $\sigma_{\mu, i}$, the chi square of the $\mathrm{SN}$ Ia measurement is given by

$$
\chi_{\mathrm{SN} \mathrm{Ia}}^{2}=\sum_{i} \frac{\left[\mu_{\mathrm{obs}}\left(z_{i}\right)-\mu_{\mathrm{th}}\left(z_{i}\right)\right]^{2}}{\sigma_{\mu, i}^{2}},
$$

where $\mu_{\mathrm{th}}\left(z_{i}\right)$ is the theoretical value of $\mu\left(z_{i}\right)$ known for a given dark energy model.

The position of the CMB acoustic peaks is determined by the following parameter $43-45$ ]

$$
l_{a}=\frac{\pi d_{a}^{(c)}\left(z_{*}\right)}{r_{s}\left(z_{*}\right)}
$$

where $z_{*}$ is the redshift at the decoupling epoch, $d_{a}^{(c)}\left(z_{*}\right)=\mathcal{R} /\left(H_{0} \sqrt{\Omega_{m 0}}\right)$ is the comoving angular diameter distance to the last scattering surface $\left(\Omega_{m 0}\right.$ is the matter density parameter today), and

$$
\mathcal{R}=\sqrt{\Omega_{m 0}} \int_{0}^{z_{*}} \frac{d z}{H(z) / H_{0}} .
$$

The sound horizon $r_{s}\left(z_{*}\right)$ is defined by

$$
r_{s}\left(z_{*}\right)=\int_{z_{*}}^{\infty} \frac{d z}{H(z) \sqrt{3\left\{1+3 \Omega_{b 0} /\left[4 \Omega_{\gamma 0}(1+z)\right]\right\}}} .
$$

Here $\Omega_{b 0}$ and $\Omega_{\gamma 0}$ are the today's density parameters of baryons and photons, respectively. For the redshift $z_{*}$ we use the fitting formula of $\mathrm{Hu}$ and Sugiyama [46]. ${ }^{4}$ The chi square associated with the WMAP7 measurement is

$$
\chi_{\mathrm{CMB}}^{2}=\boldsymbol{X}_{\mathrm{CMB}}^{T} \boldsymbol{C}_{\mathrm{CMB}}^{-1} \boldsymbol{X}_{\mathrm{CMB}},
$$

where $\boldsymbol{X}_{\mathrm{CMB}}^{T}=\left(l_{a}-302.09, \mathcal{R}-1.725, z_{*}-1091.3\right)$, and the covariance matrix is given by [4]

$$
\boldsymbol{C}_{\mathrm{CMB}}=\left(\begin{array}{ccc}
0.58269 & 0.00274801 & 0.318613 \\
0.00274801 & 0.000338358 & 0.0122901 \\
0.318613 & 0.0122901 & 0.824753
\end{array}\right),
$$

and the inverse covariance matrix is

$$
\boldsymbol{C}_{\mathrm{CMB}}^{-1}=\left(\begin{array}{ccc}
2.305 & 29.698 & -1.333 \\
29.698 & 6825.27 & -113.18 \\
-1.333 & -113.18 & 3.414
\end{array}\right) .
$$

In $\mathrm{BAO}$ observations the ratio $r_{\mathrm{BAO}}(z) \equiv$ $r_{s}\left(z_{d}\right) / D_{V}(z)$ is measured, where $r_{s}\left(z_{d}\right)$ is the sound

\footnotetext{
4 We note that, for fixed $l_{a}$, two parameters $\mathcal{R}$ and $z_{*}$ depend primarily on $\Omega_{m 0}$ and $\Omega_{b 0}$, respectively. While $l_{a}$ characterizes the position of the CMB acoustic peaks, the parameters $\Omega_{m 0}$ and $\Omega_{b 0}$ are mostly related to the amplitudes of the peaks [46]. It is possible to employ the parameter sets $\left(l_{a}, \Omega_{m 0}, \Omega_{b 0}\right)$ in the likelihood analysis (as in Ref. [44]), but we use the parameter sets $\left(l_{a}, \mathcal{R}, z_{*}\right)$ because those are the parameters that the WMAP team provides an approximate covariance matrix for.
}

horizon at which the baryons are released from the Compton drag of photons and $D_{V}(z)$ is the effective BAO distance defined by $D_{V}(z) \equiv\left[\left(\int_{0}^{z} H^{-1}(\tilde{z}) d \tilde{z}\right)^{2} z / H(z)\right]^{1 / 3}$ 5]. For the redshift $z_{d}$ we use the fitting formula of Eisenstein and $\mathrm{Hu}$ [47]. The chi square of the SDSS7 measurement is given by

$$
\chi_{\mathrm{BAO}, \mathrm{SDSS} 7}^{2}=\boldsymbol{X}_{\mathrm{BAO}}^{T} \boldsymbol{C}_{\mathrm{BAO}}^{-1} \boldsymbol{X}_{\mathrm{BAO}},
$$

where $\boldsymbol{X}_{\mathrm{BAO}}^{T}=\left(r_{\mathrm{BAO}}(0.2)-0.1905, r_{\mathrm{BAO}}(0.35)-0.1097\right)$. The covariance matrix is given by $[\underline{6}]$

$$
\boldsymbol{C}_{\mathrm{BAO}}=\left(\begin{array}{cc}
3.7436 \times 10^{-5} & 7.4148 \times 10^{-6} \\
7.4148 \times 10^{-6} & 1.2966 \times 10^{-5}
\end{array}\right),
$$

and the inverse covariance matrix is

$$
\boldsymbol{C}_{\mathrm{BAO}}^{-1}=\left(\begin{array}{cc}
30124 & -17227 \\
-17227 & 86977
\end{array}\right) .
$$

We also use the BAO data from the WiggleZ and 6dFGS surveys, for which $A_{\text {WiggleZ }}(z=0.6)=0.452 \pm 0.018$ [48], and $A_{6 \mathrm{dFGS}}(z=0.106)=0.526 \pm 0.028$ [49], where $A(z)$ is defined as $A_{\text {th }}(z)=D_{V}(z) \sqrt{\Omega_{m 0} H_{0}^{2}} / z$.

Finally, we use the latest and most precise data point from the BOSS experiment [29], for which

$$
1 / r_{\mathrm{BAO}}(z=0.57)=13.67 \pm 0.22 .
$$

Note that the error bar of this data is less than $1.7 \%$. This is the most precise distance measurement ever constrained from a galaxy survey. Moreover, as we will see below, this data puts a severe upper bound of $w$ close to -1 at $z=0.57$.

The total chi-square from the three datasets is

$$
\chi^{2}=\chi_{\mathrm{SN} \mathrm{Ia}}^{2}+\chi_{\mathrm{CMB}}^{2}+\chi_{\mathrm{BAO}}^{2},
$$

where the best-fit corresponds to the lowest value of $\chi^{2}$.

\section{A. Thawing models}

Let us study observational constraints on thawing models given by the equation of state (8). Since $K$ is weakly constrained in the three-parameter analysis, we first study this model by fixing the value of $K$ and vary the two parameters $w_{0}$ and $\Omega_{\phi 0}$. This is the approach taken by Dutta and Scherrer [25]. However, because of a mild dependence (i.e. degeneracy) of the $\chi^{2}$ as a function of the parameter $K$, we will also perform the data analysis by marginalizing the $\chi^{2}$ over $K$ itself. This last procedure allows us, by hiding the information of $K$, to understand the measured parameters $\Omega_{\phi 0}$ and $w_{0}$ easily.

Although quintessence corresponds to the case $w_{0}>$ -1 , we also extend to the regime $w_{0}<-1$ in the likelihood analysis. In fact it was shown in Ref. [50] that the equation of state of a phantom scalar field can be accommodated by the analytic formula (8). The likelihood 
results are quite different depending on whether the recent BAO data from BOSS 29] are included or not, so we present two constraints with/without the BOSS data.

Let us first focus our analysis on some particular values of $K$, which allow us to make comparison with the results given in 25]. Our numerical analysis gives that, in the absence of the BOSS BAO data, the dark energy equation of state today is constrained to be $-1.219<w_{0}<-0.930$ (at $95 \% \mathrm{CL}$ for $K=1.01$ ). Dutta and Scherrer 25] showed that even the value $w_{0}=-0.7$ is allowed from the SN Ia data alone. Adding the WMAP7 and SDSS7 data gives rise to much tighter bounds on $w_{0}$. This is the case for $\Omega_{\phi 0}$ as well. We obtained the bound $0.7078<$ $\Omega_{\phi 0}<0.740$ (95\% CL for $K=1.01$ ), whereas the values $0.68<\Omega_{\phi 0}<0.8$ are allowed in Ref. [25].

We also study the same case with the latest BOSS data. Having so far fixed $K$, by studying the $\chi^{2}$ over the parameter space of the remaining two parameters, we find that $w_{0}$ and $\Omega_{\phi 0}$ are constrained to be $-1.242<$ $w_{0}<-0.995$ and $0.705<\Omega_{\phi 0}<0.734$, respectively, at $95 \% \mathrm{CL}$ for $K=1.01$. Rather surprisingly, the allowed parameter space in the regime $w_{0} \geq-1$ is very narrow. In particular the $\Lambda$ CDM model, which corresponds to $w_{0}=-1$, is outside the $1 \sigma$ observational contour. This comes from the fact that the BOSS data (32) does not allow a large parameter space with $w>-1$ at the redshift $z=0.57$.

We have also studied the case $K=4$ and derived bounds for $w_{0}$ and $\Omega_{\phi 0}$. Without the BOSS data we obtain the bounds $-1.408<w_{0}<-0.855$ and $0.711<$ $\Omega_{\phi 0}<0.740$ (95\% CL, $K=4$ ), whereas with the BOSS data these parameters are constrained to be $-1.460<$ $w_{0}<-0.982$ and $0.709<\Omega_{\phi 0}<0.734(95 \% \mathrm{CL}, K=4)$ respectively. In the former case the upper bound of $w_{0}$ gets larger than that for $K=1.01$. This reflects the fact that $w$ changes more rapidly at late times for larger $K$. In the presence of the BOSS data, however, the allowed parameter space in the regime $w_{0}>-1$ is still very tiny. This same qualitative behavour holds for all the different values of $K$ considered in our analysis, as we have found that the $\chi^{2}$ does not vary significantly as a function of $K$.

We also vary the three parameters $w_{0}, \Omega_{\phi 0}$, and $K$ in the likelihood analysis with the prior $0.1<K<10$ updating the analysis made in Ref. [28]. We have fixed this prior for $K$ because, for $K>10$, the analytic expression (8) is not completely reliable because of the rapid rolling down of the field along the potential with carefully chosen initial conditions. With the BOSS data taken into account, the best-fit model parameters are found to be $w_{0}=-1.102, \Omega_{\phi 0}=0.71955$, and $K=0.1$ with $\chi_{\min }^{2}=568.57$. Under the prior $w_{0} \geq-1$ the best-fit parameters reduce to those in the $\Lambda \mathrm{CDM}$ model as $\chi^{2}$ has a minimum at $w_{0}=-1$.

Although the case $w_{0}<-1$ is plagued by a ghost problem, the dynamics of $w$ given phenomenologically by Eq. (8) is able to fit the data quite well for $w_{0} \approx-1.2$. In fact, with two parameters more than those in the

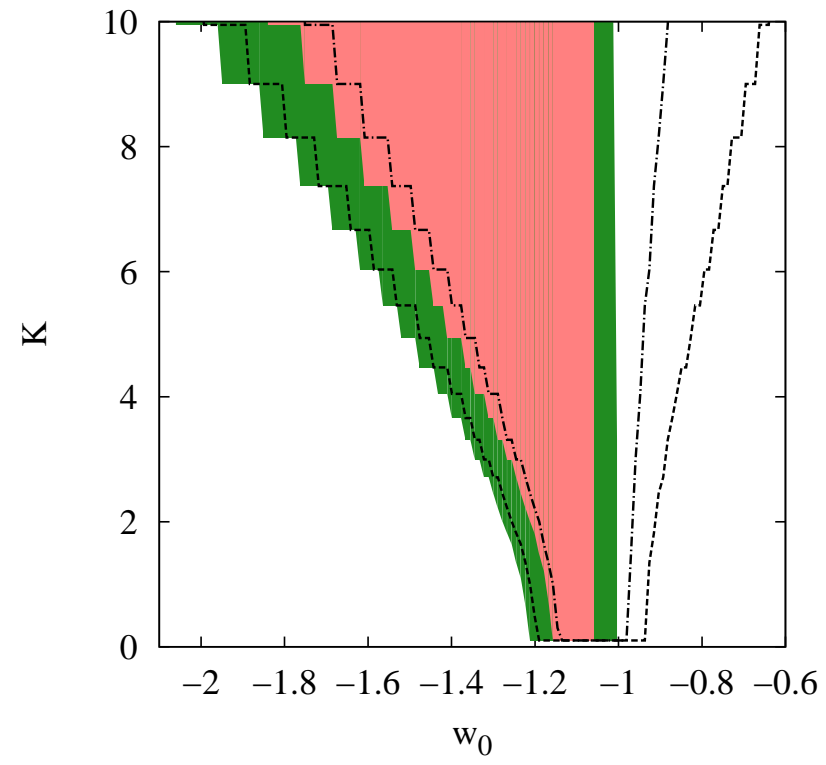

Figure 4: $1 \sigma$ (red) and $2 \sigma$ (green) observational contours in the $\left(w_{0}, K\right)$ plane marginalized over $\Omega_{\phi 0}$. We set the prior $0.1<K<10$. The dot-dashed and dotted curves correspond to the $1 \sigma$ and $2 \sigma$ constraints without the BOSS data, respectively.

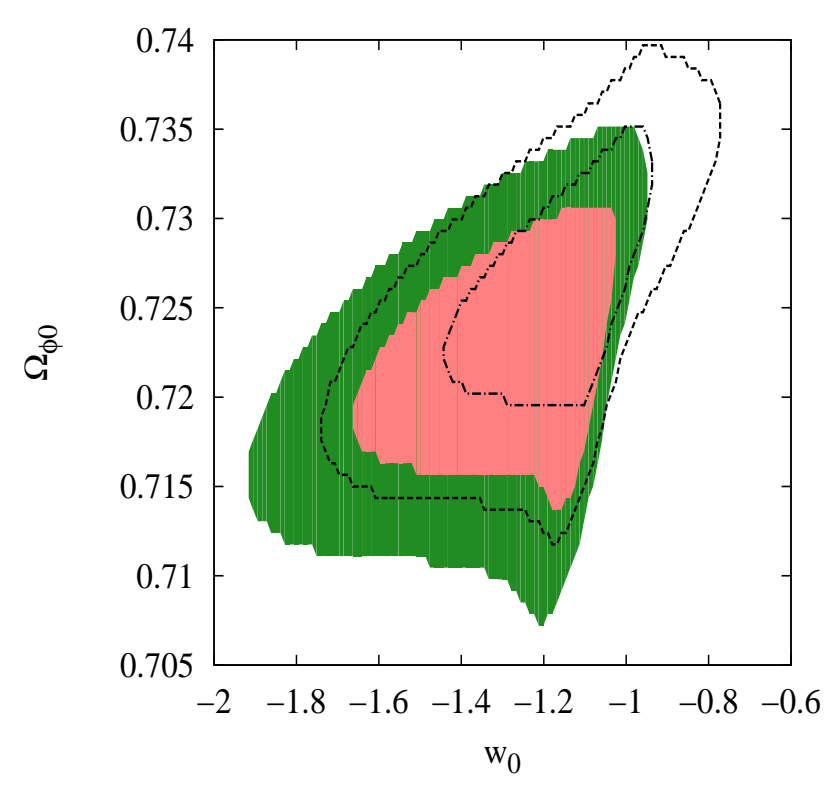

Figure 5: $1 \sigma$ (red) and $2 \sigma$ (green) observational contours in the $\left(w_{0}, \Omega_{\phi 0}\right)$ plane marginalized over $K$. We set the prior $0.1<K<10$. The dot-dashed and dotted curves correspond to the $1 \sigma$ and $2 \sigma$ constraints without the BOSS data, respectively.

$\Lambda \mathrm{CDM}$, according to the Akaike Information Criterion (AIC) (where we should add twice the number of free 
parameters $k$ to the original $\left.\chi^{2}\right)[\underline{51}]^{5}$, the best-fit corresponds to $\tilde{\chi}^{2}=568.57+2 \times 3=574.57$, whereas in the $\Lambda \mathrm{CDM} \tilde{\chi}_{\Lambda \mathrm{CDM}}^{2}=573.89+2=575.89$. Therefore the model with $w_{0}<-1$, even with three parameters, can compete with the $\Lambda$ CDM.

In Fig. 4 we show observational constraints in the $\left(w_{0}, K\right)$ plane marginalized over $\Omega_{\phi 0}$. In the regime $0.1<K<1$ the constraints on $w_{0}$ are practically independent of $K$, i.e., $-1.212<w_{0}<-1.003$ (95\% CL) with the BOSS data, which should be compared with the previous result of Ref. [33] (slightly updating the result in Ref. [28]),$-1.14<w_{0}<-0.92$ for $K<2$. In the presence of the BOSS data the allowed region shifts toward $w$ less than -1 , as we see in Fig. 4.

For $K>1$ the lower bound on $w$ gets smaller with increasing $K$, whereas the upper bound on $w$ is practically unchanged. If $K=9.95$, for example, $w_{0}$ is constrained to be $-2.059<w_{0}<-1.014(95 \% \mathrm{CL})$. For $K$ larger than the order of 1 the field equation of state can rapidly increase in low redshifts, but such rapid growth of $w$ is strongly disfavored from the BOSS data. If we do not take into account the BOSS data, the growth of $w$ away from $w_{0}=-1$ can be still allowed. For $K>10$ the analytic expression (8) is not completely reliable because of the rapid rolling down of the field along the potential with carefully chosen initial conditions.

Finally, in Fig. 5 we plot observational constraints in the $\left(w_{0}, \Omega_{\phi 0}\right)$ plane marginalized over $K$ with the prior $0.1<K<10$. Also in this case, we find the same trend already mentioned above, namely, in the presence of the BOSS data, the allowed region shifts toward the values of $w$ less than -1 , as we see in Fig. 5 . After the marginalization over $K$ we obtain the bounds $-2.18<w_{0}<-0.893$ and $0.70265<\Omega_{\phi 0}<0.73515(95 \% \mathrm{CL})$. If we put the prior $w_{0}>-1$, we find that $w_{0}$ is constrained to be $w_{0}<-0.849(68 \% \mathrm{CL})$ and $w_{0}<-0.695(95 \% \mathrm{CL})$.

\section{B. Tracking freezing models}

Let us proceed to observational constraints on tracker solutions whose equation of state is given by Eq. (18). Although $w_{(0)}$ is theoretically larger than -1 for quintessence, we do not put prior $w_{(0)} \geq-1$ in the actual likelihood analysis. In Fig. 6 we show $1 \sigma$ and $2 \sigma$ observational contours in the $\left(w_{(0)}, \Omega_{\phi 0}\right)$ plane.

Without the BAO data, the tracker equation of state is constrained to be $-1.188<w_{(0)}<-0.949(95 \%$ CL). Meanwhile the analysis of Ref. [33] based on the Constitution SN Ia and the SDSS BAO data gives the bound $-1.19<w_{(0)}<-0.90(95 \%$ CL). Hence the

\footnotetext{
${ }^{5}$ AIC assumes the infinite number of data points. For finite number of data points $(n)$, AIC should be modified to the Sugiura's criterion: $\chi^{2}+2 n k /(n-k-1)$, where $k$ is the number of free parameters [52]. Since in our case $n$ is large $(n>500)$ and $k$ is of the order of 1 , the difference between two criteria is very small.
}

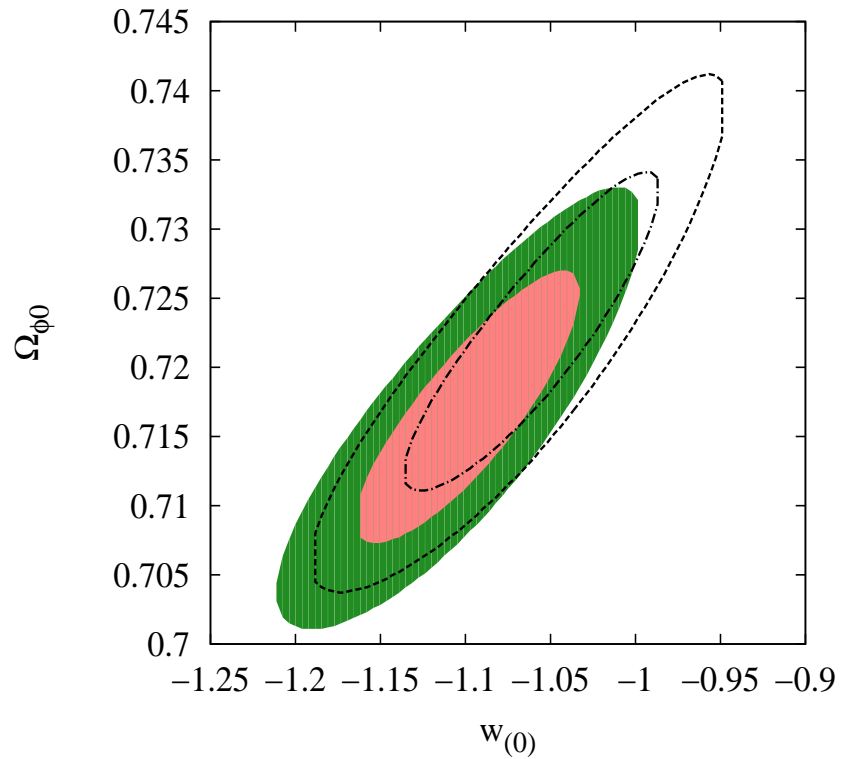

Figure 6: $1 \sigma$ (red) and $2 \sigma$ (green) observational contours on tracking freezing models in the $\left(w_{(0)}, \Omega_{\phi 0}\right)$ plane. The dotdashed and dotted curves correspond to the $1 \sigma$ and $2 \sigma$ constraints without the BOSS data, respectively.

upper bound of $w_{(0)}$ becomes tighter by including the WMAP7 data of CMB shift parameters. For the potential $V(\phi)=M^{4+p} \phi^{-p}(p>0)$ the $2 \sigma$ constraint $w_{(0)}<-0.949$ translates into $p<0.107$.

If we take into account the BOSS data in the analysis, the best-fit model parameters are found to be $w_{(0)}=$ -1.097 , and $\Omega_{\phi 0}=0.717$ with $\chi_{\min }^{2}=568.39$. In this case the Akaike criterion gives $\tilde{\chi}^{2}=568.39+2 \times 2=$ 572.39 , which is smaller than the $\Lambda \mathrm{CDM}$ value $\tilde{\chi}_{\Lambda \mathrm{CDM}}^{2}=$ 575.89 with the difference more than 2 . The $2 \sigma$ observational bounds are found to be $-1.211<w_{(0)}<-0.998$ and $0.701<\Omega_{\phi 0}<0.733$ (95\% CL). The upper bound of $w_{(0)}$ is very close to -1 , which shows that the tracking quintessence away from -1 is strongly disfavored from the data.

If we put the prior $w_{(0)} \geq-1$ in the analysis with the BOSS data, we find that the best-fit is obtained for $w_{(0)}=-1$ and the model coincides with the standard $\Lambda \mathrm{CDM}$ case. We then obtain the upper bound $w_{(0)}<$ -0.964 (95\% CL).

\section{Scaling freezing models}

For the scaling models characterized by the potential (19) with $\lambda_{2}=0$ we already showed in Sec. IIC that the evolution of $w$ can be well approximated by Eq. (20). This parametrization admits an exact solution of $H / H_{0}$ in terms of $a$ and the other free four parameters, which is numerically convenient for analyzing the data [40]. In Fig. 7 we plot observational bounds in the $\left(a_{t}, \Omega_{m 0}\right)$ plane derived by using the parametrization (20) with 


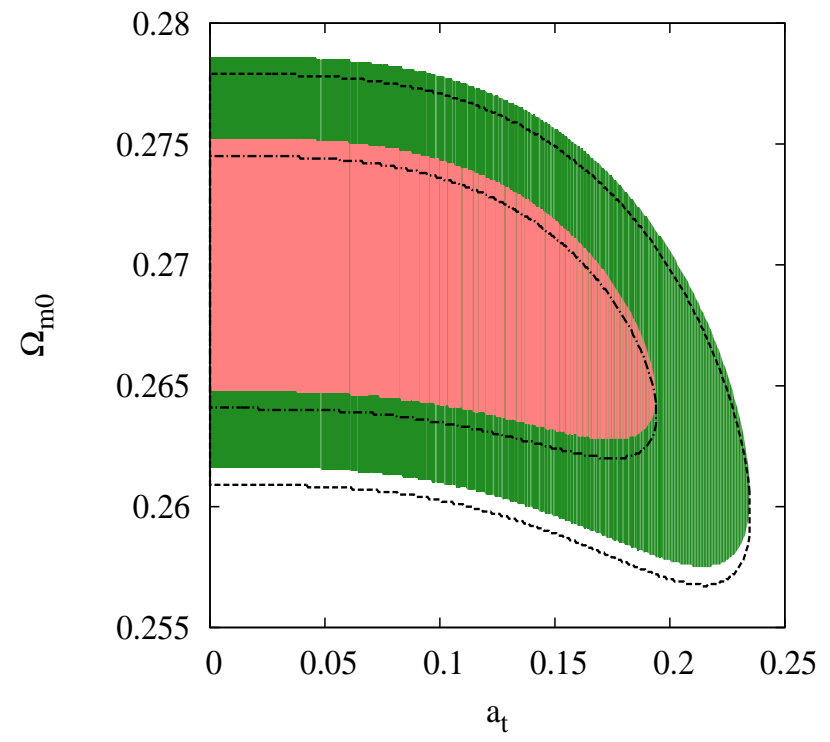

Figure 7: $1 \sigma$ (red) and $2 \sigma$ (green) observational contours on $a_{t}$ and $\Omega_{m 0}$ for the parametrization (20) of the scaling solution with $w_{p}=0, w_{f}=-1$, and $\tau=0.33$. The dot-dashed and dotted curves correspond to the $1 \sigma$ and $2 \sigma$ constraints without the BOSS data, respectively.

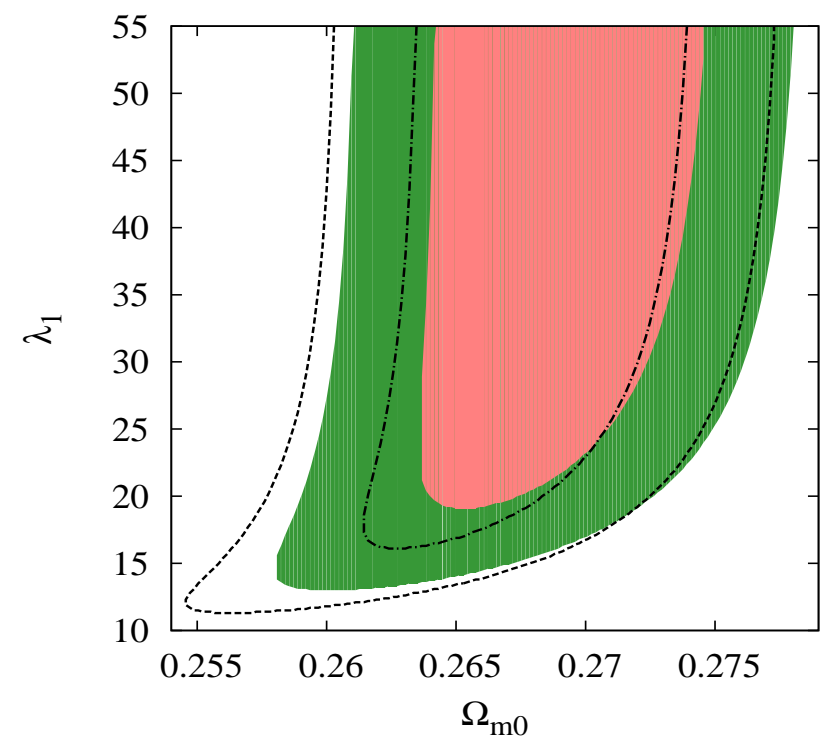

Figure 8: $1 \sigma$ (red) and $2 \sigma$ (green) observational contours on $\lambda_{1}$ and $\Omega_{m 0}$ for the potential (19) with $\lambda_{2}=0$. The dotdashed and dotted curves correspond to the $1 \sigma$ and $2 \sigma$ constraints without the BOSS data, respectively.

$w_{p}=0, w_{f}=-1$, and $\tau=0.33$. If the BOSS data are taken into account in the analysis, the transition redshift is constrained to be $a_{t}<0.23(95 \% \mathrm{CL})$. The minimum of $\chi^{2}$ is found to be $a_{t}=0$ with $\Omega_{m 0}=0.27$, i.e., the $\Lambda$ CDM limit. The case (a) shown in Fig. 2 corresponds to the marginal one in which the model is within the $2 \sigma$ observational contour. This means that $w$ needs to approach -1 in an early cosmological epoch $(w<-0.8$ for

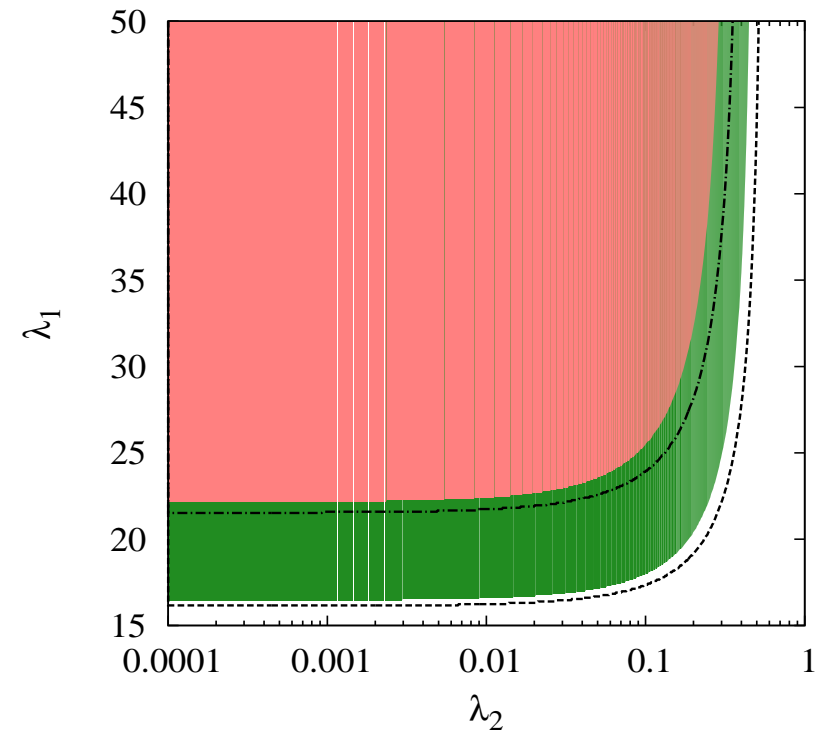

Figure 9: $1 \sigma$ (red) and $2 \sigma$ (green) observational contours on $\lambda_{1}$ and $\lambda_{2}$ for the potential (19) with $\Omega_{m 0}=0.269$. The dot-dashed and dotted curves correspond to the $1 \sigma$ and $2 \sigma$ constraints without the BOSS data, respectively.

the redshift $z<2$ ). Even without the BOSS data the upper bound on $a_{t}$ is practically unchanged, which reflects the fact that $w$ is close to -1 at low redshifts.

For $\lambda_{2}=0$ we also carry out the likelihood analysis without resorting to the parametrization (20). Numerically we solve the background equations of motion by tuning initial conditions to find the evolution which gives the desired values of $\Omega_{m 0}$ and $\Omega_{r 0}$ (radiation density parameter) for the potential $V(\phi)=V_{1} e^{-\lambda_{1} \phi / M_{\mathrm{pl}}}+V_{2}$. We put the prior $\lambda_{1}>9.4$ coming from the constraint of big bang nucleosynthesis. As long as $V_{1} e^{-\lambda_{1} \phi / M_{\mathrm{pl}}} \gg V_{2}$ the solutions approach the scaling fixed point $x=y=$ $\sqrt{6} /\left(2 \lambda_{1}\right)$ during the matter era, so the initial conditions of $x$ and $y$ are irrelevant to the likelihood analysis. In Fig. 8 we show observational bounds on the parameters $\lambda_{1}$ and $\Omega_{m 0}$. The parameter $\lambda_{1}$ is constrained to be $\lambda_{1}>13(95 \% \mathrm{CL})$, which is consistent with the results presented in Fig. 7 For larger $\lambda_{1}$ the transition from $w=0$ to $w=-1$ occurs earlier, so that the models are favored from the data.

For non-zero values of $\lambda_{2}$ we do not have an analytic expression of $w$, so we solve the background equations of motion numerically with the priors $\lambda_{1}>9.4$ and $\lambda_{2}>10^{-4}$. We set the latter prior because the $\lambda_{2}=0$ case was already discussed above. Varying the three parameters $\lambda_{1}, \lambda_{2}$, and $\Omega_{m 0}$, we find that the best-fit model parameters are $\lambda_{1}=54.94, \lambda_{2}=10^{-4}$, and $\Omega_{m 0}=0.269$ with $\chi_{\min }^{2}=574.18$. Then the Akaike criterion gives $\tilde{\chi}^{2}=574.18+2 \times 3=580.18$, which is larger than the $\Lambda \mathrm{CDM}$ value $\tilde{\chi}_{\Lambda \mathrm{CDM}}^{2}=575.89$ with the difference more 
than 4 . We also obtain the following bounds

$$
\begin{aligned}
0.262 & <\Omega_{m 0}<0.276 \quad(68 \% \mathrm{CL}), \\
0.256 & <\Omega_{m 0}<0.279 \quad(95 \% \mathrm{CL}), \\
\lambda_{1} & >16.3 \quad(68 \% \mathrm{CL}), \\
\lambda_{1} & >11.7 \quad(95 \% \mathrm{CL}), \\
\lambda_{2} & <0.361 \quad(68 \% \mathrm{CL}), \\
\lambda_{2} & <0.539 \quad(95 \% \mathrm{CL}) .
\end{aligned}
$$

Figure 9] shows the observational constraints in the $\left(\lambda_{1}, \lambda_{2}\right)$ plane for $\Omega_{m 0}=0.269$. If the three parameters $\lambda_{1}, \lambda_{2}, \Omega_{m 0}$ are varied in the likelihood analysis, it is difficult to marginalize over $\Omega_{m 0}$ in the range $0<\Omega_{m 0}<1$ because the solutions are prone to numerical instabilities around the tail regions of $\Omega_{m 0}$. Hence we use the fixed density parameter $\Omega_{m 0}=0.269$, which corresponds to the best-fit value when the three parameters are varied.

If $\lambda_{2} \gtrsim 0.5$, the model is excluded at $95 \% \mathrm{CL}$. This is associated with the fact that $w$ possesses a minimum for larger $\lambda_{2}$ (see Fig. 3). Therefore, in the context of this model, the expansion of the Universe accelerates forever. If $\lambda_{1}=20$, for example, the parameter $\lambda_{2}$ is constrained to be $\lambda_{2} \lesssim 0.3(95 \% \mathrm{CL})$. In fact the minimum of $w$ appears for $\lambda_{2} \gtrsim 0.3$, which leads to the deviation of $w$ from -1 today. In summary, the model is within the $2 \sigma$ observational contour provided that $\lambda_{2} \lesssim 0.1$ and $\lambda_{1} \gg 1$.

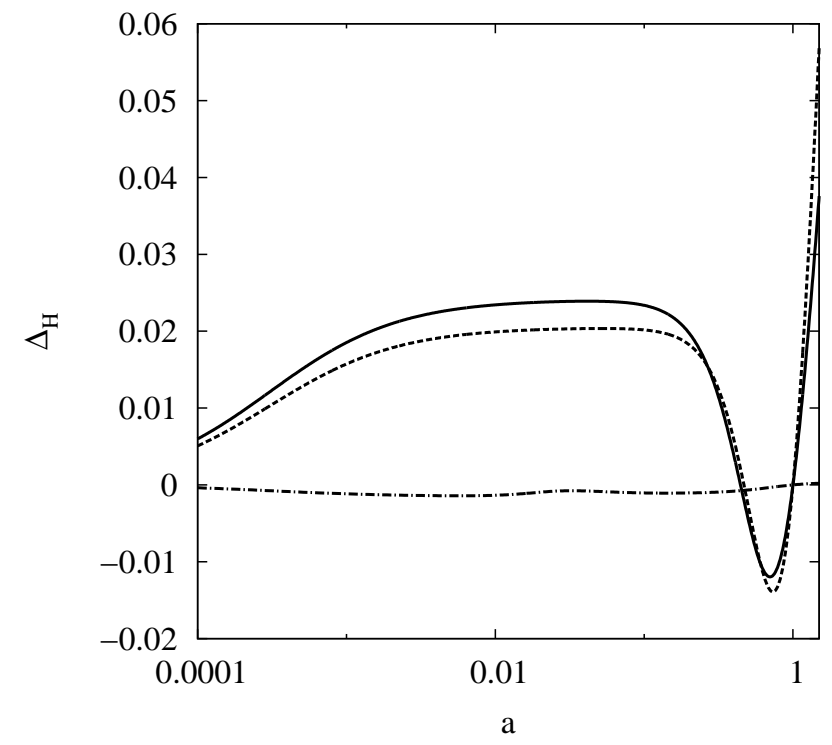

Figure 10: Relative deviation of the Hubble parameter, $\Delta_{H} \equiv$ $\left(H-H_{\Lambda \mathrm{CDM}}\right) / H_{\Lambda \mathrm{CDM}}$, for the best-fit cases of: (i) scaling (dot-dashed line), (ii) thawing (dotted line), (iii) tracker (solid line) models. The relative deviations from the $\Lambda \mathrm{CDM}$ model are less than $2.5 \%$ for $a \leq 1$. The difference in the evolution among these models during dust domination, is due to the fact that different model parameters lead to different values of $\Omega_{m 0}$ for the best fit (in the $\Lambda$ CDM we have $\Omega_{m 0}=0.2699$, whereas the $\Omega_{m 0}$ 's for the other models exceed this value by about $4 \%$ ).
In Fig. 10, we plot the evolution of the relative deviation of the Hubble parameter from the $\Lambda$ CDM as a function of $a$ for three best-fit models studied in this paper. The deviation $\Delta_{H}$ of the best-fit scaling model is very small, which reflects the fact that $w$ quickly approaches -1 after the early transition from the scaling regime. Meanwhile, in the best-fit thawing and tracker models, the relative deviations from the $\Lambda \mathrm{CDM}$ can reach the level of $2 \%$.

\section{CONCLUSIONS}

In this paper we placed observational bounds on three types of quintessence models: (i) thawing, (ii) tracker, and (iii) scaling models. We used the recent data of SN Ia from Union 2.1, the CMB shift parameters from WMAP7, and BAO from SDSS7 and BOSS, by which the background cosmic expansion history is constrained from the distance measurements.

In thawing models where the field starts to evolve at the late cosmological epoch, the dark energy equation of state can be expressed as Eq. (8) with the three parameters $w_{0}, \Omega_{\phi 0}$, and $K$. The parameter $K$ is related to the field mass at the initial stage. We put observational bounds in the $\left(w_{0}, \Omega_{\phi 0}\right)$ after marginalizing over $K$ (see Fig. 51. If we take into account the BOSS data (32) in the likelihood analysis, the upper bounds on $w_{0}$ are very close to -1 independent of the values of $K$ ranging in the region $0.1<K<10$ (under which the analytic formula (8) is trustable). Without the BOSS data the deviation of $w$ away from -1 today can be still allowed, as seen in Fig. 5 .

In tracking freezing models where $w$ is nearly constant $\left(w \approx w_{(0)}\right)$ during the matter era, there is the analytic formula (18) derived by considering a homogeneous perturbation around the tracker. Without the BOSS data the tracker equation of state is constrained to be $-1.188<w_{(0)}<-0.949$ (95\% CL), whose upper bound is tighter than $w_{(0)}<-0.90$ derived by using SN Ia and SDSS7 data 33. Inclusion of the BOSS data gives the upper bounds of $w_{(0)}$ close to -1 . We find that without the prior on $w_{(0)}$ the constraint is $-1.211<$ $w_{(0)}<-0.998(95 \% \mathrm{CL})$ and with the quintessence prior $w_{(0)}>-1$ the upper bound is $w_{(0)}<-0.964(95 \% \mathrm{CL})$.

For the potential (19) with $\lambda_{1} \gg 1$ and $\lambda_{2} \ll 1$, w is close to 0 during the deep matter era because of the dominance of the steep potential $V_{1} e^{-\lambda_{1} \phi / M_{\mathrm{pl}}}$. The field equation of state starts to decrease after the potential $V_{2} e^{-\lambda_{2} \phi / M_{\mathrm{pl}}}$ dominates over $V_{1} e^{-\lambda_{1} \phi / M_{\mathrm{pl}}}$. For larger $\lambda_{1}$ the exit from the scaling regime $(w=0)$ occurs earlier. When $\lambda_{2}=0$ we found that the evolution of $w$ can be approximated by the parametrization (20) with $w_{p}=$ $0, w_{f}=-1$, and $\tau \simeq 0.33$, where the transition scale factor $a_{t}$ depends on $\lambda_{1}$. Using this parametrization we derived the bound $a_{t}<0.23(95 \% \mathrm{CL})$, which translates into the constraint $\lambda_{1}>13$. This is consistent with the bound shown in Fig. 7 derived by solving the background equations of motion numerically. 
For the potential (19) with non-zero values of $\lambda_{2}$, the field equation of state tends to have a minimum for larger $\lambda_{2}$. As we see in Fig. 9, the models with $\lambda_{2} \gtrsim 0.5$ is excluded at $95 \% \mathrm{CL}$. The parameters $\lambda_{2} \lesssim 0.1$ and $\lambda_{1}>17$ are allowed from the data, which shows that the early transition from the scaling regime to the regime close to $w=-1$ is favored. For the scaling models the observational constraints on $\lambda_{1}$ and $\lambda_{2}$ are not very sensitive to inclusion of the BOSS data, because $w$ evolves toward -1 at the late cosmological epoch.

As we see in Fig. 10, the difference of $H$ between the three best-fit quintessence models and the $\Lambda$ CDM model is only less than $2.5 \%$ in the past. In current observations there is no strong evidence that quintessence is favored over the $\Lambda C D M$ from the statistical point of view. This property is especially significant by including the BOSS BAO data.

If we extend the analysis in the regime $w<-1$, we showed that some of the models studied in this paper can compete with the $\Lambda$ CDM model according to the Akaike information criterion. We note that in many modified gravity models such as $f(R)$ gravity [53], (extended) Galileons [54], and Lorentz violating theories [55] it is possible to realize $w<-1$ without having ghosts and instabilities. It remains to see how much extent future high-precision observations can constrain dark energy models from the background expansion history as well as from the cosmic growth of density perturbations.

\section{ACKNOWLEDGEMENTS}

AD thanks JSPS for financial support to visit Tokyo University of Science (No. S12135). ST thanks warm hospitalities during his stays in Weihai, Observatorio Nacional in Rio de Janeiro, Passa Quatro, Szczecin, and University of Heidelberg. This work was supported in part by the Grant-in-Aid for Scientific Research Fund of the JSPS No. 24540287 (TC) and No. 24540286 (ST) and Scientific Research on Innovative Areas (No. 21111006) (ST) and in part by Nihon University (TC).
[1] A. G. Riess et al. [Supernova Search Team Collaboration], Astron. J. 116, 1009 (1998); S. Perlmutter et al. [Supernova Cosmology Project Collaboration], Astrophys. J. 517, 565 (1999).

[2] M. Hicken et al., Astrophys. J. 700, 1097 (2009).

[3] D. N. Spergel et al. [WMAP Collaboration], Astrophys. J. Suppl. 148, 175 (2003).

[4] E. Komatsu et al. [WMAP Collaboration], Astrophys. J. Suppl. 192, 18 (2011).

[5] D. J. Eisenstein et al. [SDSS Collaboration], Astrophys. J. 633, 560 (2005).

[6] W. J. Percival et al., Mon. Not. Roy. Astron. Soc. 401, 2148 (2010).

[7] N. Suzuki et al., Astrophys. J. 746, 85 (2012).

[8] S. Weinberg, Rev. Mod. Phys. 61, 1 (1989).

[9] Y. Fujii, Phys. Rev. D 26, 2580 (1982); L. H. Ford, Phys. Rev. D 35, 2339 (1987); C. Wetterich, Nucl. Phys B. 302, 668 (1988); T. Chiba, N. Sugiyama and T. Nakamura, Mon. Not. Roy. Astron. Soc. 289, L5 (1997); P. G. Ferreira and M. Joyce, Phys. Rev. Lett. 79, 4740 (1997); R. R. Caldwell, R. Dave and P. J. Steinhardt, Phys. Rev. Lett. 80, 1582 (1998).

[10] P. J. E. Peebles and B. Ratra, Astrophys. J. 325, L17 (1988); B. Ratra and J. Peebles, Phys. Rev D 37, 321 (1988).

[11] T. Chiba, T. Okabe and M. Yamaguchi, Phys. Rev. D62, 023511 (2000); C. Armendariz-Picon, V. F. Mukhanov and P. J. Steinhardt, Phys. Rev. Lett. 85, 4438-4441 (2000).

[12] V. Sahni and A. A. Starobinsky, Int. J. Mod. Phys. D 9, 373 (2000); S. M. Carroll, Living Rev. Rel. 4, 1 (2001); T. Padmanabhan, Phys. Rept. 380, 235 (2003); P. J. E. Peebles and B. Ratra, Rev. Mod. Phys. 75, 559 (2003); E. J. Copeland, M. Sami and S. Tsujikawa, Int. J. Mod. Phys. D 15, 1753 (2006); T. P. Sotiriou and V. Faraoni, Rev. Mod. Phys. 82, 451 (2010); A. De Felice and S. Tsujikawa, Living Rev. Rel. 13, 3 (2010);
S. Tsujikawa, arXiv:1004.1493 [astro-ph.CO].

[13] S. M. Carroll, Phys. Rev. Lett. 81, 3067 (1998).

[14] C. F. Kolda and D. H. Lyth, Phys. Lett. B 458, 197 (1999).

[15] P. Binetruy, Phys. Rev. D 60, 063502 (1999); P. Brax and J. Martin, Phys. Lett. B 468, 40 (1999); E. J. Copeland, N. J. Nunes and F. Rosati, Phys. Rev. D 62, 123503 (2000); P. K. Townsend, JHEP 0111, 042 (2001); S. Hellerman, N. Kaloper and L. Susskind, JHEP 0106, 003 (2001); R. Kallosh, A. D. Linde, S. Prokushkin and M. Shmakova, Phys. Rev. D 65, 105016 (2002); M. Gasperini, F. Piazza and G. Veneziano, Phys. Rev. D 65, 023508 (2002); A. J. Albrecht, C. P. Burgess, F. Ravndal and C. Skordis, Phys. Rev. D 65, 123507 (2002).

[16] J. A. Frieman, C. T. Hill, A. Stebbins and I. Waga, Phys. Rev. Lett. 75, 2077 (1995).

[17] Y. Nomura, T. Watari and T. Yanagida, Phys. Lett. B 484, 103 (2000); K. Choi, Phys. Rev. D 62, 043509 (2000); J. E. Kim and H. P. Nilles, Phys. Lett. B 553, 1 (2003); L. J. Hall, Y. Nomura and S. J. Oliver, Phys. Rev. Lett. 95, 141302 (2005); S. Panda, Y. Sumitomo and S. P. Trivedi, Phys. Rev. D 83, 083506 (2011).

[18] R. R. Caldwell and E. V. Linder, Phys. Rev. Lett. 95, 141301 (2005).

[19] I. Zlatev, L. M. Wang and P. J. Steinhardt, Phys. Rev. Lett. 82, 896 (1999); P. J. Steinhardt, L. -M. Wang and I. Zlatev, Phys. Rev. D 59, 123504 (1999).

[20] E. J. Copeland, A. R. Liddle and D. Wands, Phys. Rev. D 57, 4686 (1998).

[21] T. Barreiro, E. J. Copeland and N. J. Nunes, Phys. Rev. D 61, 127301 (2000).

[22] A. Albrecht and C. Skordis, Phys. Rev. Lett. 84, 2076 (2000).

[23] V. Sahni and L. -M. Wang, Phys. Rev. D 62, 103517 (2000).

[24] T. D. Saini, S. Raychaudhury, V. Sahni and 
A. A. Starobinsky, Phys. Rev. Lett. 85, 1162 (2000); Z. Haiman, J. J. Mohr and G. P. Holder, Astrophys. J. 553, 545 (2000); C. Baccigalupi, A. Balbi, S. Matarrese, F. Perrotta and N. Vittorio, Phys. Rev. D 65, 063520 (2002); M. Yahiro, G. J. Mathews, K. Ichiki, T. Kajino and M. Orito, Phys. Rev. D 65, 063502 (2002); U. Alam, V. Sahni, T. D. Saini and A. A. Starobinsky, Mon. Not. Roy. Astron. Soc. 344, 1057 (2003); R. R. Caldwell and M. Doran, Phys. Rev. D 69, 103517 (2004); L. Perivolaropoulos, Phys. Rev. D 71, 063503 (2005) S. Nesseris and L. Perivolaropoulos, Phys. Rev. D 72, 123519 (2005); D. Huterer and H. V. Peiris, Phys. Rev. D 75, 083503 (2007); M. Sahlen, A. R. Liddle and D. Parkinson, Phys. Rev. D 75, 023502 (2007); S. Sen, A. A. Sen and M. Sami, Phys. Lett. B 686, 1 (2010); S. Thakur, A. Nautiyal, A. A. Sen and T. R. Seshadri, arXiv:1204.2617 [astro-ph.CO].

[25] S. Dutta and R. J. Scherrer, Phys. Rev. D 78, 123525 (2008).

[26] T. Chiba, Phys. Rev. D 79, 083517 (2009).

[27] R. J. Scherrer and A. A. Sen, Phys. Rev. D 77, 083515 (2008).

[28] T. Chiba, S. Dutta and R. J. Scherrer, Phys. Rev. D 80, 043517 (2009).

[29] L. Anderson et al., arXiv:1203.6594 [astro-ph.CO].

[30] N. G. Busca et al. [BOSS Collaboration], arXiv:1211.2616 [astro-ph.CO].

[31] T. Clemson and A. R. Liddle, Mon. Not. Roy. Astron. Soc. 395, 1585 (2009).

[32] G. Gupta, S. Majumdar and A. Sen, Mon. Not. Roy. Astron. Soc. 420, 1309 (2012).

[33] T. Chiba, Phys. Rev. D 81, 023515 (2010).

[34] C. R. Watson and R. J. Scherrer, Phys. Rev. D 68, 123524 (2003).

[35] P. - Y. Wang, C. -W. Chen and P. Chen, JCAP 1202, 016 (2012).

[36] A. de la Macorra and G. Piccinelli, Phys. Rev. D 61, 123503 (2000); S. C. C. Ng, N. J. Nunes and F. Rosati, Phys. Rev. D 64, 083510 (2001).

[37] T. Chiba, Phys. Rev. D 66, 063514 (2002).

[38] F. Piazza and S. Tsujikawa, JCAP 0407, 004 (2004); S. Tsujikawa and M. Sami, Phys. Lett. B 603, 113 (2004).
[39] R. Bean, S. H. Hansen and A. Melchiorri, Phys. Rev. D 64, 103508 (2001).

[40] E. V. Linder and D. Huterer, Phys. Rev. D 72, 043509 (2005).

[41] B. A. Bassett, M. Kunz, J. Silk and C. Ungarelli, Mon. Not. Roy. Astron. Soc. 336, 1217 (2002); P. S. Corasaniti and E. J. Copeland, Phys. Rev. D 67, 063521 (2003); B. A. Bassett, P. S. Corasaniti and M. Kunz, Astrophys. J. 617, L1 (2004).

[42] A. De Felice, S. Nesseris and S. Tsujikawa, JCAP 1205, 029 (2012).

[43] J. R. Bond, G. Efstathiou and M. Tegmark, Mon. Not. Roy. Astron. Soc. 291, L33 (1997).

[44] A. Kosowsky, M. Milosavljevic and R. Jimenez, Phys. Rev. D 66, 063007 (2002).

[45] Y. Wang and P. Mukherjee, Phys. Rev. D 76, 103533 (2007).

[46] W. Hu and N. Sugiyama, Astrophys. J. 471, 542 (1996).

[47] D. J. Eisenstein and W. Hu, Astrophys. J. 496, 605 (1998).

[48] C. Blake et al., Mon. Not. Roy. Astron. Soc. 415, 2892 (2011).

[49] F. Beutler et al., Mon. Not. Roy. Astron. Soc. 416, 3017 (2011).

[50] S. Dutta, E. N. Saridakis and R. J. Scherrer, Phys. Rev. D 79, 103005 (2009).

[51] H. Akaike, IEEE Trans. Auto. Control, 19, 716 (1974); A. R. Liddle, Mon. Not. Roy. Astron. Soc. 351, L49 (2004).

[52] N. Sugiura, Communications in Statistics, A7, 13-26 (1978).

[53] W. Hu and I. Sawicki, Phys. Rev. D 76, 064004 (2007); L. Amendola and S. Tsujikawa, Phys. Lett. B 660, 125 (2008); H. Motohashi, A. A. Starobinsky and J. 'i. Yokoyama, Prog. Theor. Phys. 123, 887 (2010).

[54] A. De Felice and S. Tsujikawa, Phys. Rev. Lett. 105, 111301 (2010); A. De Felice and S. Tsujikawa, JCAP 1202, 007 (2012).

[55] V. A. Rubakov, Theor. Math. Phys. 149, 1651 (2006); M. Libanov, V. Rubakov, E. Papantonopoulos, M. Sami and S. Tsujikawa, JCAP 0708, 010 (2007). 